\title{
Optimal surgical timing and approach for tibial plateau fracture
}

\author{
Kai Li ${ }^{\mathrm{a}, 1}$, Shuang Zhang ${ }^{\mathrm{a}, \mathrm{b}, \mathrm{c}, \mathrm{d}, \mathrm{e}, 1}$, XuZhong Qiu ${ }^{\mathrm{a}}$, HaiJun Huang ${ }^{\mathrm{a}}$, Hao Sheng ${ }^{\mathrm{a}}$, Yun Zhang ${ }^{\mathrm{a}}$, \\ Jihui Chang ${ }^{\mathrm{a}, *}$, Jiangming Kuang ${ }^{\mathrm{b}}$ and Jining Yang ${ }^{\mathrm{b}}$ \\ ${ }^{a}$ Department of Orthopaedics, People's Hospital of Zhongjiang County, Zhongjiang, Sichuan, China \\ ${ }^{\mathrm{b}}$ Data Recovery Key Laboratory of Sichuan Province, School of Artificial Intelligence, Neijiang Normal \\ University, Neijiang, Sichuan, China \\ ${ }^{\mathrm{c}}$ School of Life Science and Technology, University of Electronic Science and Technology of China, \\ Chengdu, Sichuan, China \\ ${ }^{\mathrm{d}}$ High Field Magnetic Resonance Brain Imaging Laboratory of Sichuan, University of Electronic Science \\ and Technology of China, Chengdu, Sichuan, China \\ ${ }^{\mathrm{e}}$ Key Laboratory for Neuroinformation, Ministry of Education, University of Electronic Science and \\ Technology of China, Chengdu, Sichuan, China
}

\begin{abstract}
.
BACKGROUND: Tibial plateau fracture is a common fracture encountered in the clinic.

OBJECTIVE: This study determined the optimal timing and surgical approach for patients with tibial plateau fracture.

METHODS: Fifty-two patients with complex tibial plateau fractures were treated in our hospital (the People's Hospital of Zhongiiang County) between 2013 and 2015. These patients were recruited as participants in this study; all patients were randomly allocated into two groups of 26 patients each. Patients in Group 1 underwent single-incision, single-plate knee surgeries via an antero-lateral approach, and patients in Group 2 underwent anterior median incisions of the knee for double-plate surgeries. The effects of the approaches were compared and analyzed.

RESULTS: The best time to perform surgery was 6-8 days post-injury. The anterior median incision, double-plate method approach was better than the antero-lateral, single-incision, single-plate method. For the former method, the healing among middle-aged and young patients was better than that of elderly patients, and that healing of men was slightly better than that of female patients. However, the degree of healing among patients was $>80 \%$ at 5 months postoperatively. The purpose of surgical management has been fully achieved.

CONCLUSION: The optimal timing of surgery for patients with complex tibial plateau fractures is $6-8$ days post-injury. The surgical approach needs to be determined based on the actual condition of the patient. However, the treatment effect of an anterior median incision, double-plate method is better, and the recovery rate may approach $80 \%$ at 5 months postoperatively.
\end{abstract}

Keywords: Tibial plateau fracture, surgical timing, operative mode, statistics, imageology

\section{Introduction}

The knee joint is the most complex joint and an important load-bearing joint of the lower limbs [1-3]. As one of the main bony structures of this joint, the tibial plateau plays a very important role. Tibial

\footnotetext{
${ }^{1}$ These authors contributed equally to this work.

${ }^{*}$ Corresponding author: Jihui Chang, Department of Orthopaedics, People's Hospital of Zhongjiang County, Zhongjiang, Sichuan 6181001, China. E-mail: 442644280@qq.com.
} 
plateau fractures account for approximately $1.7 \%$ of all human fractures [4,5], and approximately $18.6 \%$ of tibial fractures. It is a common type of fracture encountered in the clinic [6]. However, fractures involving the tibial plateau have complex manifestations, and because the tibial plateau and the proximal tibia have important positions in the knee joint structure, treatment of these fractures is challenging.

The tibial plateau is located at the metaphysis of the proximal tibia. With an expanded shape, it is conducive to knee joint stability. Furthermore, more muscles, tendons, and ligaments are attached to it. Its cancellous bone is rich, dense bone is thin, and its ability to resist traumatic injury is poor. Above the joint is the articular surface of the tibial plateau, which corresponds to the articular surface of the femoral condyle. The medial plateau is large and concave, while the lateral plateau is small and convex, slightly higher than the medial articular surface. The medial condyle is firmer than the lateral condyle; hence, the lateral plateau is more prone to collapse and fragmentation, and the medial platform is more prone to massive fracture, often accompanied by more serious injury and even dislocation $[4,5]$. Fractures of the tibial plateau may cause uneven forces on the medial plateau and the lateral plateau of the knee joint, resulting in further bone and joint disease, thereby threatening the patient's health [4]. The clinical treatment of complex tibial plateau fracture has always been the focus of clinical attention and research as far as orthopedics is concerned. In the treatment process, we need to consider both treatment of tibial fracture and the avoidance of damage to the cartilaginous structures. If the treatment is improper, the disability rate these types of fractures is very high.

In the recent years, discussions on tibial plateau fractures have gradually increased. Some scholars have found that at least $7 \%$ of tibial plateau fractures include posterior column fractures [7]. Patients with tibial plateau fractures treated by surgery have lower incidence of vascular and nerve injury. Their knee-joint range of motion is significantly improved, and their incidence of incision complications is also lower. Furthermore it is easier to achieve anatomical reduction with surgical repair. However, for different types of patients, there is a lack of systematic research on when it is best to perform the surgical intervention, surgical methods, and postoperative effects. This study aimed to determine the optimal timing and surgical approach for patients with tibial plateau fracture.

\section{Data and methods}

\subsection{General data}

Fifty-two patients with tibial plateau fractures admitted to the People's Hospital of Zhongjiang County between January 2013 and December 2015 were recruited as research participants. All patients were randomly divided into the following two groups:

Group 1: Twenty-six patients, including 15 males and 11 females; the average age of the patients was $(42.31 \pm 2.16)$ years;

Group 2: Twenty-six patients, 16 males and 10 females; the average age of the patients was $(42.25 \pm$ 2.33) years;

The two groups of patients had no significant differences in terms of sex, age, and other basic information $(P>0.05)$; hence, they were comparable.

Inclusion criteria [8-11]: (1) Both groups of patients underwent imaging examinations before participating in this study, and the examination results conformed to the relevant diagnostic criteria for tibial plateau fractures. (2) Before participating in this study, the doctors introduced the basic premise of this study and the potential problems that may arise from its conduct to the patients. Due attention was paid towards protecting the privacy of all patients. After listening to the introduction, the patients all agreed 
that the doctors would use the case data, and the patients themselves or their family members signed an informed consent form. This study was approved by the hospital ethics department and met all relevant medical research standards.

\subsection{Treatment method}

Group 1: The patients' knees were treated using an anterolateral single-incision approach and a singleplate method. General anesthesia was administered following endotracheal intubation, with the patient in the supine position. An air bag tourniquet was applied on the upper thigh. A $15-\mathrm{cm}$ surgical incision was made from the upper edge of the lateral tibia to the lower part of the tibial tubercle, to expose the anterior tibial muscle and the articular surface of the lateral tibial plateau. The $\mathrm{C}$-arm machine was used for X-ray fluoroscopy; the Kirschner wire was used for reduction and fixation; and the L-shaped locking plate was placed on the outside of the tibia.

Group 2: The knees of these patients were treated with an anterior median incision double-plate method: anesthesia, position, and use of tourniquet were the same as those in Group 1. An 18-cm surgical incision was reckoned from the anterolateral suprapatellar - anterior spine of upper middle tibia, the sub periosteum was properly stripped, and the fractured end of medial tibial plateau was fully exposed. The fracture site was reduced and fixed with plates and screws, and the surgical scheme of lateral tibia was the same as that in Group 1.

\subsection{Observation indicators}

(1) The incidence of complications in patients based on timing of surgical intervention (0-3 days after injury, 4-5 days after injury, 6-8 days after injury, and $>9$ days after injury) were compared and analyzed.

(2) The intraoperative blood loss, fracture healing time, internal fixation failure rate, and surgical site infection rate of the two groups were recorded accordingly; the data of the two groups were compared and analyzed.

\subsection{Data analysis}

The statistical software SPSS version 18.0 (IBM, Armonk, New York, United States of America) was used for data analysis and processing; $(\bar{x} \pm s)$ and $(\%)$ were used to represent measurement and counting-type indicators. The differences between the two indicators were tested by independent samples $t$ and $x^{2}$, respectively. A $P$ value $<0.05$ was deemed statistically significant.

\section{Results}

\subsection{Analysis of statistical results}

\subsubsection{Surgical timing and incidence of complications}

To study the incidence of complications caused by varying amounts of time after injury, a statistical analysis of the operations performed at different times post-injury and the incidence of complications was performed, as shown in Table 1.

The incidence of complications among patients who underwent surgery 4-5 days post-injury is lower than that of patients who underwent surgery within 3 days following injury $(P<0.05)$. The incidence of complications among patients undergoing surgery $6-8$ days post injury is lower than that of patients undergoing the procedure $0-5$ days and $>9$ days after injury $(P<0.05)$. 
Table 1

Comparison of complication incidence at different surgical timing $[n(\%)]$

\begin{tabular}{ccccc}
\hline \multicolumn{1}{c}{ Time } & Skin ischemic necrosis & Superficial infection & Deep infection & Complication incidence \\
\hline $0-3 \mathrm{~d}(n=7)$ & $2(28.57)$ & $1(14.29)$ & $1(14.29)$ & 57.15 \\
$4-5 \mathrm{~d}(n=10)$ & $1(10)$ & $1(10)$ & $1(10)$ & 30 \\
$6-8 \mathrm{~d}(n=30)$ & $0(0)$ & $1(3.33)$ & $0(0)$ & 3.33 \\
$\geqslant 9 \mathrm{~d}(n=5)$ & $1(20)$ & $0(0)$ & $0(0)$ & 20 \\
\hline
\end{tabular}

${ }^{*} n$ : complication incidence.

Table 2

Control analysis

\begin{tabular}{lcccc}
\hline \multicolumn{1}{c}{ Group } & $\begin{array}{c}\text { Intraoperative blood } \\
\text { loss }(\mathrm{ml})\end{array}$ & Healing time (d) & $\begin{array}{c}\text { Internal fixation } \\
\text { failure rate }\end{array}$ & $\begin{array}{c}\text { Surgical site } \\
\text { infection }\end{array}$ \\
\hline Observation group $(n=26)$ & $268.74 \pm 54.23$ & $158.67 \pm 21.36$ & $5(19.23)$ & $2(7.69)$ \\
Control group $(n=26)$ & $325.98 \pm 52.35$ & $121.25 \pm 20.61$ & $1(3.85)$ & $2(7.69)$ \\
$t / x^{2}$ & 3.462 & 5.638 & 4.157 & 0.000 \\
$P$ & 0.001 & 0.000 & 0.042 & 1 \\
\hline
\end{tabular}

* Observation Group: Group1, Control Group: Group 2.

\subsubsection{Control analysis}

To analyze the therapeutic effects of the two different methods on patients, we conducted a controlled study considering aspects of blood loss, healing time, internal fixation failure rate, and surgical site infection. The detailed results are shown in Table 2.

The healing time and internal fixation failure rate of the fractures of Group 2 were significantly better than those of Group 1, while the intraoperative blood loss of Group 1 was less than that of Group $2(P<$ $0.05)$. At the same time, there was no significant difference in surgical site infection rate between the two groups $(P>0.05)$. From the perspective of achieving surgical cure, the plan implemented for Group 2 was significantly better than that for Group 1 .

\subsection{Imageology analysis}

To analyze the healing effect of tibial plateau fracture patients with plate screws for reduction and fixation of the fracture site, we randomly selected four patients (two males and two females) from Group 2 to conduct an imageology study. The four patients represented different ages, different degrees of injury, and different sexes. Patients 1 and 2 were young and middle-aged (aged between 35 and 50 years), of which patient 1 was male and patient 2 was female. Patients 3 and 4 were elderly (aged between 55 and 68 years), of which patient 3 was male and patient 4 was female. The results are shown in Fig. 1.

According to the imaging data (Fig. 1) of four patients, the degree of injury for patients 1 and 4 was basically the same, and that of patients 2 and 3 was basically the same. However, the injury intensity of patients 2 and 3 is significantly greater than that of patients 1 and 4 (pre-operative image), resulting in increased surgical complexity for patients 2 and 3 in comparison to that of patients 1 and 4 (post-operative image). Hence, their risk of incurring postoperative infection is greater and the probability of complete recovery is less. Per re-examination of imaging 5 months postoperatively, considering the same intensity of injury, after they were managed using plates and screws for reduction and fixation the fractured site, the recovery of young and middle-aged patients (Patients 1 and 2) was significantly better than that of elderly patients (Patients 3 and 4). At the same time, concerning recovery from local injury, we also found that recovery from injury among male patients was slightly better than that of female patients. This could inform the development of surgical and medical strategies in the future. Judging from the imaging data of 


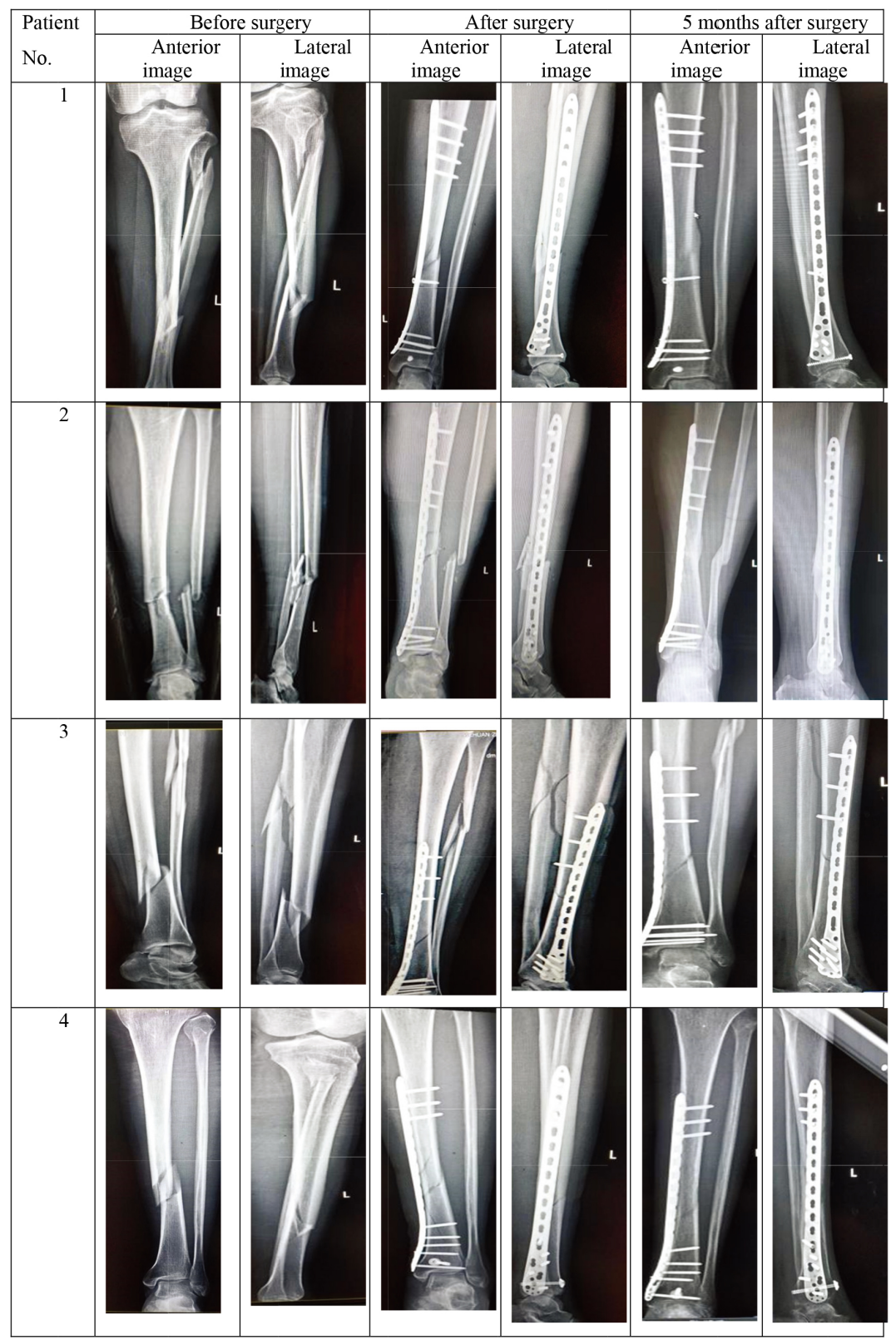

Fig. 1. Operative strategy and subsequent effects.

all patients before surgery and 5 months after surgery, the degree of recovery of all patients in 5 months postoperatively was $>80 \%$, indicating that the treatment goal was fully achieved.

\section{Conclusions}

In this study, the surgical timing and surgical methods used for patients with complex tibial plateau fractures were studied. From the results of statistical analysis, the incidence of complications in patients 
undergoing surgery 6-8 days after injury was the lowest, while the incidence of complications in patients undergoing surgery 3 days after injury was the highest. From the selection of surgical methods and treatment effect, patients in Group 1 who underwent anterolateral single-incision and single-plate management of the affected knee had less intraoperative blood loss. Compared with group 1, the healing time of fractures in Group 2 who were treated with the double-plate method and approached with anterior median incision of the affected knee was shorter. The failure rate of internal fixation was also lower $(P<$ $0.05)$ with this procedure; however, there was no significant difference $(P>0.05)$ between the two groups in the incision infection rate. This result is consistent with the results of related studies [5,6,9-11]. The statistical results fully proved that the optimal timing of surgery for patients with complex tibial plateau fractures was 6-8 days post-injury, although the surgical method needed to be deliberated based on the actual situation of the patient, the anterior median incision, double-plate method had better therapeutic outcomes. Similarly, through imaging studies of patients treated with the knee anterior median incision double-plate method and combined with demographic studies, it was seen that the recovery of young and middle-aged patients at 5 months post-operatively was significantly better than that for elderly patients. Additionally, in the analysis of postoperative local images, it was also found that the recovery of male patients from injury was slightly better than that compared with female patients of the same age, which may inform surgical plans and medical management in the future. This increases the effective rate of clinical management and will improve the quality of life of patients.

\section{Acknowledgments}

The work presented in this paper is supported by the Foundation of Ph.D. Scientific Research of Neijiang Normal University (Grant 18B19), the Sichuan Applied Psychology Research Center of Chengdu Medical College Funded Projects (Grant CSXL-21103), and the Innovative Team Program of the Neijiang Normal University (Grant 2021TD02).

\section{Conflict of interest}

The authors declare no conflict of interest.

\section{References}

[1] Tran J, Peng PWH, Chan VWS, Agur AMR, Overview of innervation of knee joint, Physical Medicine and Rehabilitation Clinics of North America, 2021, 32(4): 767-778.

[2] Hatfield GL, Costello KE, Wilson JLA, Stanish WD, Hubley-Kozey CL, Association between knee joint muscle activation and knee joint moment patterns during walking in moderate medial compartment knee osteoarthritis: Implications for secondary prevention, Archives of Physical Medicine and Rehabilitation, 2021, 102(10): 1910-1917.

[3] Hummer E, Thorsen T, Weinhandl JT, Cates H, Zhang SN, Knee joint biomechanics of patients with unilateral total knee arthroplasty during stationary cycling, Journal of Biomechanics, 2021, 115: 11011.

[4] Vendeuvre T, Gaye LE, Percutaneous treatment of tibial plateau fractures, Orthopaedics \& Traumatology: Surgery \& Research, 2021, 107(1), Supplement: 102753.

[5] Mauricio KJ, Fabrício F, Rogério CB, Rafael LF, Rodrigo S, Cleber AJP, TIBIAL PLATEAU FRACTURES, Revista Brasileira de Ortopedia (English Edition), 2009, 44(6): 468-474.

[6] Born CT, Gil JA, Johnson JP, Periprosthetic tibial fractures, Journal of the American Academy of Orthopaedic Surgeons, 2018, 26(8): e167-e172. doi: 10.5435/JAAOS-D-16-00387.

[7] Van den Berg JD, Quintens L, Zhan Y, Hoekstra H, Why address posterior tibial plateau fractures? Injury, 2020, 51(12): 2779-2785. 
[8] Sim JA, Park JH, Na YG, Kim J, Lee BH, Three-column subdivision for isolated posterolateral tibial plateau fractures and perspective surgical approaches, The Knee, 2021, 33: 93-101.

[9] Van den Berg J, De Boer AS, Assink N, Haveman R, Reul M, Link BC, Ijpma FFA, Verhofstad MHJ, Hoekstra H, Trauma mechanism and patient reported outcome in tibial plateau fractures with posterior involvement, The Knee, 2021, 30: 41-50.

[10] Elsoe R, Larsen P, Delayed but favourable outcome of lateral tibial plateau fracture after screw fixation: A 3-year prospective cohort study of 56 patients, The Knee, 2021, 29: 280-290.

[11] Elsoe R, Motahar I, Mahdi F, Larsen P, Presence of magnetic resonance imaging verified soft tissue injuries did not significantly affect the patient-reported outcome 12 months following a lateral tibial plateau fracture: A 12-month prospective cohort study of 56 patients, The Knee, 2020, 27(2): 420-427. 